\title{
An unusual presentation of Behçet's disease with inferior vena cava thrombosis and pulmonary embolism
}

\author{
Karunanayaka SK, Jayawardhane VV, Premawansa G \\ North Colombo Teaching Hospital, Ragama, Sri Lanka. \\ Correspondence: Dr. Salika Karunanayaka \\ e-mail: salikalana@gmail.com \\ https://orcid.org/0000-0002-4257-6556 \\ Submitted on 27.11.2020 and accepted for publication on 13.06.2021
}

\section{Introduction}

Behçet's disease (BD) is a variable vasculitis which can affect vessels of all sizes and types. It commonly affects superficial veins and is triggered by environmental factors and infections in predisposed individuals $(1,2)$. It is more prevalent among silk route descendants $(1,3)$. The Classical triad of Behçet's syndrome are oral ulcers, genital ulcers and eye involvement (1). But it can involve multi-organ systems including central nervous system, eyes, joints, gastrointestinal system and respiratory system $(1,3,4)$.

Initial presentation with inferior vena cava (IVC) thrombosis that extend up to the right atrium causing pulmonary embolism is rare (5). Thrombus formation in the vessels is due to inflammatory process causing the endothelial damage. Therefore, the thrombus is tightly adherent to the vessel wall. It makes the pulmonary thromboembolism extremely rare. In this case report we present a rare occurrence of IVC thrombosis extending up to the right atrium and concurrent occurrence of pulmonary embolism.

\section{Case presentation}

A 46-year-old male presented with cough for 4 months, loss of appetite and loss of weight for 2 months duration. He had recurrent oral ulcers and papular pustular lesions in the body for 1 year duration and scrotal ulcers for 3 months duration. He was investigated for pyrexia of unknown origin 2 months back and was treated as having pyelonephritis on the right side, but urine culture was negative then. One scrotal ulcer had healed with scarring. At the presentation to us, he had dilated superficial abdominal veins which were draining upward after emptying with mild ankle oedema, suggestive of inferior vena cava obstruction. Following day two of the admission, he developed haemoptysis and shortness of breath categorized as grade II according to modified Medical Research Council dyspnoea (mMRC) scale. Inflammatory markers were elevated (CRP $39.4 \mathrm{mg} / \mathrm{dL}$, ESR $122 \mathrm{~mm} / \mathrm{hr}$ ). Coagulation studies were normal (INR 1.33, APTT 29.1 seconds). USS abdomen showed mild splenomegaly and IVC thrombus extending up to right atrium which was confirmed by contrast enhanced-CT of abdomen (Figure 1). Trans thoracic echo was normal and there was no thrombus formation within the right atrium. Computed Tomography Pulmonary Angiogram (CTPA) revealed a filling defect at the bifurcation of right pulmonary artery to lower lobe of the right lung and multiple filling defects were noted in the segmental branches of the pulmonary arteries of both lungs suggestive of pulmonary embolism (Figure 2). There were no arterial thickening and aneurysm formation. Sputum AFB and Mantoux were negative. Retroviral studies and HSV studies were negative. Reactivity of skin to needle prick or injection (Pathergy test) was negative (2). All the tumour markers were negative including tPSA, AFP, CA 19.9 and alpha-fetoproteins. Antiphospholipid antibodies were negative. Serum protein electrophoresis did not reveal a monoclonal band. The patient was diagnosed as having BD according to International Criteria for Behçet's Disease (ICBD) (Table 1). 


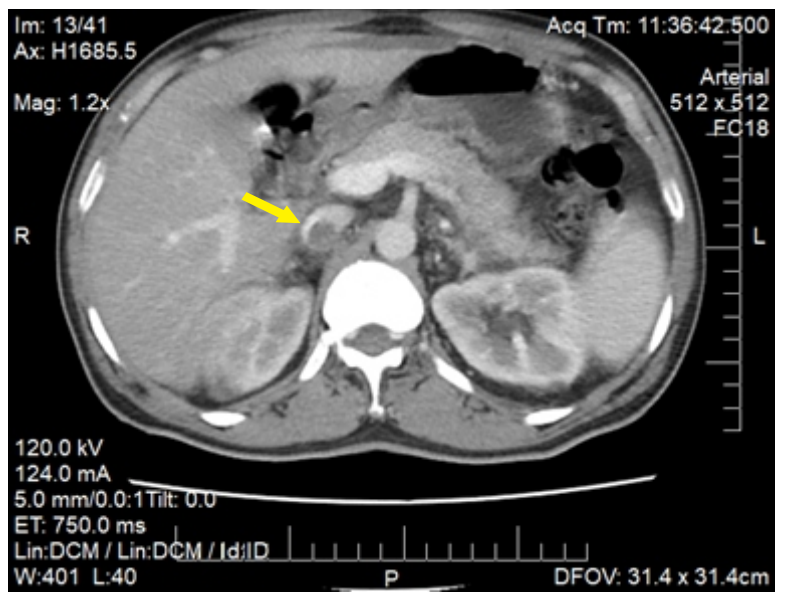

Figure 1: Contrast enhanced $\mathrm{CT}$ abdomen revealed IVC thrombus (arrow)

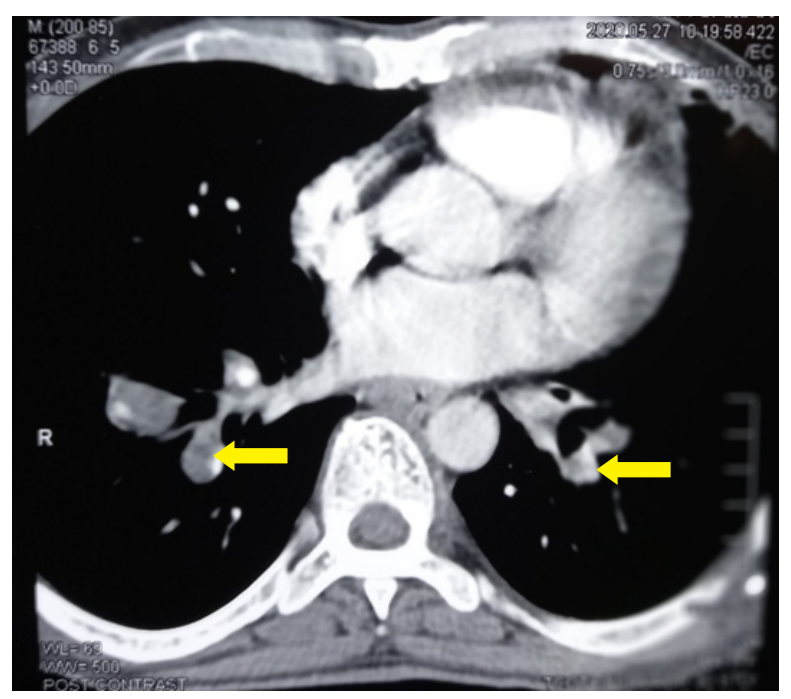

Figure 2: Multiple filling defects (arrows) in CTPA

From day 3 of admission, warfarin $5 \mathrm{mg}$ daily dose was started with the target INR of 2 to 3 to prevent the propagation of the thrombus. Subcutaneous enoxaparin $40 \mathrm{mg}$ daily dose was started simultaneously and continued till the target INR was achieved. Induction of immunosuppression was done by IV methyl prednisolone $500 \mathrm{mg}$ daily from day 5 of admission for 3 days and followed by oral prednisolone $1 \mathrm{mg} / \mathrm{kg}$ /day. Intravenous (IV) cyclophosphamide $500 \mathrm{mg} 2$ weekly pulse therapy was started from day 8 of admission. The patient was discharged after 3 weeks of hospital stay and reviewed 2 weekly till the completion of 5 cycles of IV cyclophosphamide pulses. Then, a daily dose of azathioprine $25 \mathrm{mg}$ was added and oral prednisolone was gradually tailed off over next 3 months. Repeat imaging of the abdomen following 3 months of diagnosis revealed the complete resolution of IVC thrombus. Oral anticoagulation was withheld after completion of 3 months. Patient is clinically well on oral prednisolone $10 \mathrm{mg}$ daily and azathioprine $50 \mathrm{mg}$ daily, without further relapse of the illness.

Table 1: International Criteria for Behçet's diseasepoint score system (ICBD): scoring 4

\begin{tabular}{lc}
\hline Sign / Symptom & Points \\
\hline Oral aphthosis & 2 \\
Genital aphthosis & 2 \\
Ocular lesions & 2 \\
Skin lesions & 1 \\
Vascular manifestations & 1 \\
Neurological manifestations & 1 \\
Positive pathergy test & \\
* Pathergy test is optional and the primary scoring system does \\
not include pathergy testing. However, where pathergy testing is \\
conducted one extra point may be assigned for a positive result.
\end{tabular}

\section{Discussion}

Our patient was investigated for pyrexia of unknown origin and had been treated for right side culture negative pyelonephritis, two months back, which may be the triggering factor for his disease manifestation. He had recurrent oral ulcers and he fulfilled other two criteria of BD including genital ulcers and recurrent papular pustular skin lesions (2). According to ICBD, he had 6 points with genital aphthosis, oral aphthosis, skin lesion and vascular manifestations which made him a certain case of BD (2). Though BD can affect any size and any type of vessel, it commonly affects superficial veins (3). In our patient there was IVC thrombosis which extended up to the opening of the right atrium. In $\mathrm{BD}$, thrombosis formation is due to endothelial damage and platelet aggregation (3). So, thrombus is tightly adherent to the vessel wall (5). Common pulmonary involvements are pulmonary artery aneurysms and in situ thrombus formation $(1,5)$. 
$\mathrm{BD}$ is rarely associated with pulmonary embolism (5). Anticoagulation therapy for in-situ pulmonary thrombus will lead to catastrophic sequelae with worsening of haemoptysis as thrombi are well organised due to vasculitis (3). Our patient well tolerated anticoagulation therapy which further supported our diagnosis. Tekantapeh $\mathrm{S}$ et al., reported a similar case of a patient presented with haemoptysis due to BD complicated with pulmonary embolism (5). He was also successfully managed with warfarin and enoxaparin at the acute presentation and was started on immunosuppressive and immunomodulatory treatment with prednisolone, methotrexate and cyclophosphamide monthly pulse therapy which led to a complete remission. This rare presentation highlights the importance of identification of rare presentations of the BD and starting the treatment at correct time.

Authors declare no conflicts of interest. Informed written consent was obtained from the patient for publication of this case report.

\section{Acknowledgements}

We acknowledge Rheumatology and Rehabilitation Hospital, Ragama medical team for their commitment in managing this patient.

\section{References}

1. Seyahi E, Yurdakul S. Behçet's Syndrome and Thrombosis, Mediterranean Journal of Hematology and Infectious Diseases. 2011; 3(1), e2011026. https://doi.org/10.4084/mjhid.2011.026

2. Davatchi F, Assaad-Khalil S, Calamia KT, Crook JE, Sadeghi AB, Schirmer M, Ziaei N. The International Criteria for Behçet's Disease (ICBD): A collaborative study of 27 countries on the sensitivity and specificity of the new criteria. Journal of the European Academy of Dermatology and Venereology. 2014; 28(3), 338-347.

3. Sakane T, Takeno M, Suzuki N, Inaba G. Behçet's disease. New England Journal of Medicine 1999; 341(17): 1284 1291. DOI: 10.1056/NEJM199910213411707.

4. G, Christensen R, Bang D. Update of the EULAR recommendations for the management of Behçet's syndrome, Annals of the Rheumatic Diseases. 2018; 77 : 808-818.

5. Tekantapeh S, Rashidi F, Khabbazi, A. Pulmonary Thromboembolism in Behçet's Disease: A Case Report. Rheumatology Research, 2018; 3(4): 161-164. DOI: 10.22631/rr.2018.69997.1058. 